\title{
Interdependence between electrical and magnetic properties of polycrystalline cobalt-substituted tungsten bronze multiferroic ceramics
}

\author{
Shilpi Jindal*, Sheela Devi ${ }^{\dagger}$, Ajay Vasishth ${ }^{\ddagger}$, Khalid Mujasam Batoo ${ }^{\S}$ and Gagan Kumar* \\ *Department of Physics, Chandigarh University \\ Mohali, Punjab, India \\ ${ }^{\dagger}$ Department of Applied Sciences, MSIT, C-4 \\ Janakpuri, New Delhi, India \\ *Department of Applied Sciences, Chandigarh Engineering College \\ Mohali, Punjab, India \\ $\S_{\text {King Abdullah Institute for Nanotechnology }}$ \\ King Saud University, P. O. Box 2455 \\ Riyadh 11451, Saudi Arabia \\ physics.sheela@gmail.com
}

Received 23 October 2017; Revised 11 December 2017; Accepted 3 January 2018; Published 9 February 2018

\begin{abstract}
Polycrystalline cobalt-substituted tungsten bronze ferroelectric ceramics with chemical composition $\mathrm{Ba}_{5} \mathrm{CaTi}_{2-x} \mathrm{Co}_{X} \mathrm{Nb}_{8} \mathrm{O}_{30}$ $(x=0.00,0.02,0.04$ and 0.08$)$ were synthesized by solid state reaction technique. X-ray diffraction (XRD) technique was used to confirm the phase formation and it revealed the formation of single phase tetragonal structure with space group P4bm. The surface morphology of the samples was studied by using the scanning electron microscopy (SEM) technique. The dielectric properties such as dielectric constant and dielectric loss have been investigated as a function of temperature and frequency. The $P-E$ and $M-H$ studies confirmed the coexistent of ferroelectricity and magnetism at room temperature. The $P-E$ loop study indicated an increase in the coercive field while the $M-H$ study depicted a decrease in the magnetization with the incorporation of cobalt ions.
\end{abstract}

Keywords: Tungsten bronze; solid state reaction technique: electrical properties; magnetic properties.

\section{Introduction}

Tungsten Bronze (TB) materials belong to dielectric materials which have attracted much attention in the field of material science and in the present advancement of technology because of their good dielectric, ferroelectric and nonlinear optical properties. Based on these extraordinary properties, these lead-free ferroelectric materials show a great promise in various electronic devices such as transducers, actuators, capacitors, ferroelectric random access memory (FERM) and microwave tunable device applications. ${ }^{1-5}$ The TB-type structure $^{6-9}$ consists of a complex array of distorted $\mathrm{BO}_{6}$ octahedral sharing corners in such a way that three different types of interstices (A, B and C) are available for cations' substitution in the general formula, where $A_{1}$ and $A_{2}$ sites are commonly filled by divalent or trivalent cations, $\mathrm{B}_{1}$ and $\mathrm{B}_{2}$ sites by tetravalent or pentavalent cations and $\mathrm{C}$ site being small, regularly remains vacant giving the general formula $\mathrm{A}_{6} \mathrm{~B}_{10} \mathrm{O}_{30}$. It has been found that different ionic substitutions in the above-mentioned sites can play an important role in tailoring their physical and electrical properties. So, it provides a scope for remodeling the dielectric and magnetic properties in the development of new and better materials for device applications. The detailed literature study reveals that a lot of work has been carried out on various TB ferroelectric ceramics of niobates, tantalates and vanadates having tetragonal TB (TTB) structure. Kiran et al. ${ }^{10}$ have synthesized $\mathrm{Ba}_{5} \mathrm{HoTi}_{3} \mathrm{~V}_{7} \mathrm{O}_{30}$ compound via solid state reaction and reported the electrical behavior using impedance analysis. Das et al. ${ }^{11}$ have reported the complex TB electroceramics of $\mathrm{Li}_{2} \mathrm{~Pb}_{2} \mathrm{Y}_{2} \mathrm{~W}_{2} \mathrm{Ti}_{4} \mathrm{~V}_{4} \mathrm{O}_{30}$ composition for structural and electrical properties. Ganguly et al. ${ }^{12}$ have reported the effects of calcium ions on the structural and electrical properties of $\mathrm{Ba}_{5-x} \mathrm{Ca}_{x} \mathrm{SmTi}_{3} \mathrm{Nb}_{7} \mathrm{O}_{30} \quad(x=0-5)$ ferroelectric ceramics. Chen et $a l .{ }^{13}$ have investigated the influence of $\mathrm{La}, \mathrm{Nd}, \mathrm{Sm}$ ions on the structural, dielectric and magnetic properties of $\mathrm{Ba}_{3} \mathrm{SrLn}_{2} \mathrm{Fe}_{2} \mathrm{Nb}_{8} \mathrm{O}_{30}$ TB ceramics. Yan et al. ${ }^{14}$ have analyzed the crystal structure, dielectric and magnetic properties of $\mathrm{Ba}_{5} \mathrm{NdNi}_{1.5} \mathrm{Nb}_{8.5} \mathrm{O}_{30} \mathrm{~TB}$ ceramics. Liu et al. ${ }^{15}$ have reported the effect of $\mathrm{Nb}$ substitution on structure, dielectric and ferroelectric properties of $\mathrm{Ba}_{4} \mathrm{Nd}_{2} \mathrm{Fe}_{2} \mathrm{Ta}_{8} \mathrm{O}_{30}$ ceramics. In fact, many researchers have investigated the substituted TB ceramics, but to the our best of knowledge, there is no report available on the dielectric, ferroelectric and magnetic properties of cobalt-substituted TB ceramics. Therefore, in the present work, a maiden attempt has been made to investigate the influence of cobalt ions on the structural,

This is an Open Access article published by World Scientific Publishing Company. It is distributed under the terms of the Creative Commons Attribution 4.0 (CC-BY) License. Further distribution of this work is permitted, provided the original work is properly cited. 
dielectric, ferroelectric and magnetic properties of $\mathrm{Ba}_{5} \mathrm{CaTi}_{2-x}$ $\mathrm{Co}_{x} \mathrm{Nb}_{8} \mathrm{O}_{30}$ ceramics.

\section{Experimental}

\subsection{Sample preparation}

Polycrystalline samples of composition $\mathrm{Ba}_{5} \mathrm{CaTi}_{2-x}$ $\mathrm{Co}_{x} \mathrm{Nb}_{8} \mathrm{O}_{30}(x=0.0,0.02,0.04$ and 0.08$)$ were prepared by solid state reaction method by taking high purity $\mathrm{BaCO}_{3}$, $\mathrm{CaCO}_{3}, \mathrm{CoO}, \mathrm{TiO}_{2}$ and $\mathrm{Nb}_{2} \mathrm{O}_{5}$ (all from M/S Aldrich, USA) in their stoichiometric proportions. The powder mixture was thoroughly grinded by an agate mortar for $7-8 \mathrm{~h}$ and then passed through sieve of 75 micron meter. The grind powder mixture was then calcined at $1100^{\circ} \mathrm{C}$ for $20 \mathrm{~h}$ in an alumina crucible to form the desired phase. The calcined powders were then ground and pressed at $150 \mathrm{MPa}$ into disk shaped pellets of $10 \mathrm{~mm}$ diameter and $1 \mathrm{~mm}$ thickness using $5 \mathrm{wt} . \%$ polyvinyl alcohol (M/S Aldrich, USA) as binder. The compact pellets were then sintered at $1300^{\circ} \mathrm{C}$ for $10 \mathrm{~h}$ in air.

\subsection{Characterizations}

$\mathrm{X}$-ray diffractrogram of sintered pellets were recorded by using Bruker X-ray diffractometer with $\operatorname{CuK} \alpha(\lambda=1.5406 \AA)$ radiation in the range $10^{\circ} \leq 2 \Theta \leq 80^{\circ}$ at a scanning rate of $1 \% \mathrm{~min}$. The microstructural studies of samples were carried out using scanning electron microscope (SEM) (Hitachi $\mathrm{S}-3700 \mathrm{~N}$ ). The sintered pellets were polished with silver paste on both sides and cured at $300^{\circ} \mathrm{C}$ for $1 \mathrm{~h}$. The dielectric measurements were carried out using LCR meter (Agilent 4284A) operating at oscillation amplitude of 1 V. $P-E$ hysteresis loops were recorded at room temperature using an automatic hysteresis loop tracer based on Sawyer-Tower circuit. The magnetic properties were determined by using Lake Shore's fully integrated sample magnetometer.

\section{Results and Discussions}

\subsection{Structural study}

X-Ray diffraction (XRD) patterns of composition $\mathrm{Ba}_{5} \mathrm{CaTi}_{2-x} \mathrm{Co}_{x} \mathrm{Nb}_{8} \mathrm{O}_{30}(x=0.00,0.02,0.04$ and 0.08$)$ were recorded at room temperature and are shown in Fig. 1. TBstructured compounds have either tetragonal or orthorhombic structure. XRD patterns show a large number of sharp and single diffraction peak of materials for different concentrations and were observed to have better homogeneity, crystallization as well as the formation of single phase. ${ }^{16}$ The crystallite size $D$ for the synthesized samples was calculated from the broadening of strong and medium reflection by using the Debye Scherer's equation:

$$
D=\frac{0.9 \lambda}{\beta \cos \theta}
$$

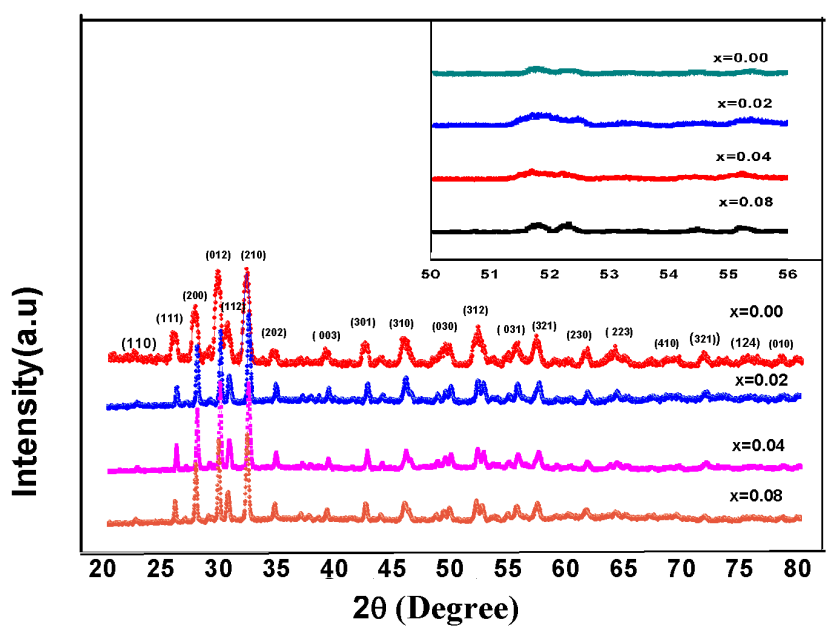

Fig. 1. XRD patterns for $\mathrm{Ba}_{5} \mathrm{CaTi}_{2-x} \mathrm{Co}_{x} \mathrm{Nb}_{8} \mathrm{O}_{30}$ ceramics (inset shows the shifting in peak with the incorporation of $\mathrm{Co}^{2+}$ ions).

where $\lambda$ is the incident wavelength, $\beta$ is full width at half maximum (FWHM) and $\theta$ is the glancing angle. The calculated values of crystallite size for all the samples of cobaltsubstituted $\mathrm{Ba}_{5} \mathrm{CaTi}_{2-x} \mathrm{Co}_{X} \mathrm{Nb}_{8} \mathrm{O}_{30}$ compound are given in Table 1 . The lattice parameters $(a, c)$ were calculated by using $\mathrm{X}$-Pert high score computer software program and the calculated values are given in Table 1 . Indexed reflections and lattice parameters indicate that the samples have tetragonal structure with space group $\mathrm{P} 4 \mathrm{bm}$. The larger ionic radii $\mathrm{Co}^{2+}$ (0.65) with respect to $\mathrm{Ti}^{2+}(0.60)$ should increase the lattice constants, thus decrease the diffraction angle $2 \theta$ as observed in Fig. 1. It can be revealed from Table 1 that the cell volume is increasing while the tetragonality $c / a$ ratio is decreasing with the substitution of cobalt ions which indicates the distortion in crystal structure.

\subsection{Stability of the crystal structure}

The stability of the TTB structure compound can be determined by Tolerance factor $(T)$ as discussed by Wakiya et al. ${ }^{17}$ According to the general formula of TTB, there are two types of A sites; one is $A_{1}$ site with 12-fold coordination which is identical as perovskite structure and another is $\mathrm{A}_{2}$ site with 15 -fold coordination which occupies pentagonal site. Thus, the value of tolerance factor $(T)$ was calculated by using the following relation: ${ }^{14}$

$$
\begin{gathered}
t_{\mathrm{A} 1}=\frac{R_{\mathrm{A} 1}+R_{\mathrm{O}}}{\sqrt{ } 2\left(R_{\mathrm{B}}+R_{\mathrm{O}}\right)}, \\
t_{\mathrm{A} 2}=\frac{R_{\mathrm{A} 2}+R_{\mathrm{O}}}{\sqrt{23-12 \sqrt{ } 3}\left(R_{\mathrm{B}}+R_{\mathrm{O}}\right)}
\end{gathered}
$$

where $t_{\mathrm{A} 1}$ and $t_{\mathrm{A} 2}$ represent the tolerance factor of $\mathrm{A}_{1}$ and $\mathrm{A}_{2}$, sites respectively, $R_{\mathrm{A}}, R_{\mathrm{B}}$ and $R_{\mathrm{O}}$ are the ionic radius of $\mathrm{A}$ sites, $\mathrm{B}$ sites and oxygen ion, respectively.

In TTB structure, tolerance factor and stability relationship analyzed by the combined relationship of the above two 
Table 1. Lattice parameter, crystalline size, lattice strain, volume and tolerance for $\mathrm{Ba}_{5} \mathrm{CaTi}_{2-x} \mathrm{Co}_{x} \mathrm{Nb}_{8} \mathrm{O}_{30}(x=0.00,0.02,0.04$ and 0.08$)$ ceramics.

\begin{tabular}{lccccccc}
\hline Sample $(\mathrm{x})$ & $a$ & $c$ & $(c / a)$ ratio & Crystallite size $(D)(\mathrm{nm})$ & Lattice strain & Volume & Tolerance factor $(T)$ \\
\hline 0.00 & 8.3311 & 12.1289 & 1.46 & 14.430 & 0.0091 & 841.83 & 0.63640 \\
0.02 & 9.9637 & 11.2176 & 1.26 & 15.080 & 0.0055 & 1113.63 & 0.63628 \\
0.04 & 10.567 & 11.1340 & 1.05 & 17.240 & 0.0050 & 1243.23 & 0.63625 \\
0.08 & 12.3064 & 9.9737 & 0.81 & 20.453 & 0.0045 & 1510.49 & 0.63619 \\
\hline
\end{tabular}

equations can be expressed as

$$
T=\frac{t_{\mathrm{A} 1}+2 t_{\mathrm{A} 2}}{3} .
$$

The crystal structure is stable when the value of $T$ is closer to 1 . The calculated values of tolerance factor $(T)$ for $\mathrm{Ba}_{5} \mathrm{CaTi}_{2-x} \mathrm{Co}_{x} \mathrm{Nb}_{8} \mathrm{O}_{30} \quad(x=0.00, \quad 0.02, \quad 0.04$ and 0.08$)$ ceramics are given in Table 1 . The observed values of tolerance factor are slightly smaller than 1 , hence showing a slight instability of the synthesized TB ceramics.

\subsection{Morphological studies}

Figure 2 shows the SEM micrographs of gold-coated specimen sintered at high temperature $1300^{\circ} \mathrm{C}$ for $10 \mathrm{~h}$. In all sintered samples of different concentrations, a well-developed poly grain with uniform grain distribution is observed. ${ }^{18}$ The average distribution of grain size for the sample was found in the range of $2-8 \mu \mathrm{m}$. Therefore, the addition of cobalt ions was observed to cause the grain growth inhibition and increase in density. The distribution of grains, size of grains and shape in the morphology have suggested that the samples have polycrystalline nature. It is clearly observed from the micrographs that site-specific substitution of cobalt ions in the TB ceramics has significant influence on the grain size and the morphology of the grains which affected the magnetic and dielectric properties. It is observed that the grain size reduced with an increase of cobalt ions up to $x<0.04$ but for $x>0.04$, an increase in the grain size was observed. The driving force which is responsible for the grain growth reduces the total grain boundary area and the interfacial free energy of grain. As the interfacial energy is inversely proportional to grain diameter, it further reduces the total grain boundary area. ${ }^{19}$ The increase grain size reduced the porosity of the material. This process is called recrystallization and depends on the high temperature sintering conditions and the concentration of the dopant.

\subsection{Dielectric study}

Figure 3 shows the temperature dependence of dielectric constant for $\mathrm{Ba}_{5} \mathrm{CaTi}_{2-x} \mathrm{Co}_{x} \mathrm{Nb}_{8} \mathrm{O}_{30}(x=0.00,0.02,0.04$ and 0.08 ) ceramics at 1,10 and $100 \mathrm{kHz}$. The dielectric constant was initially observed to increase very slowly with the
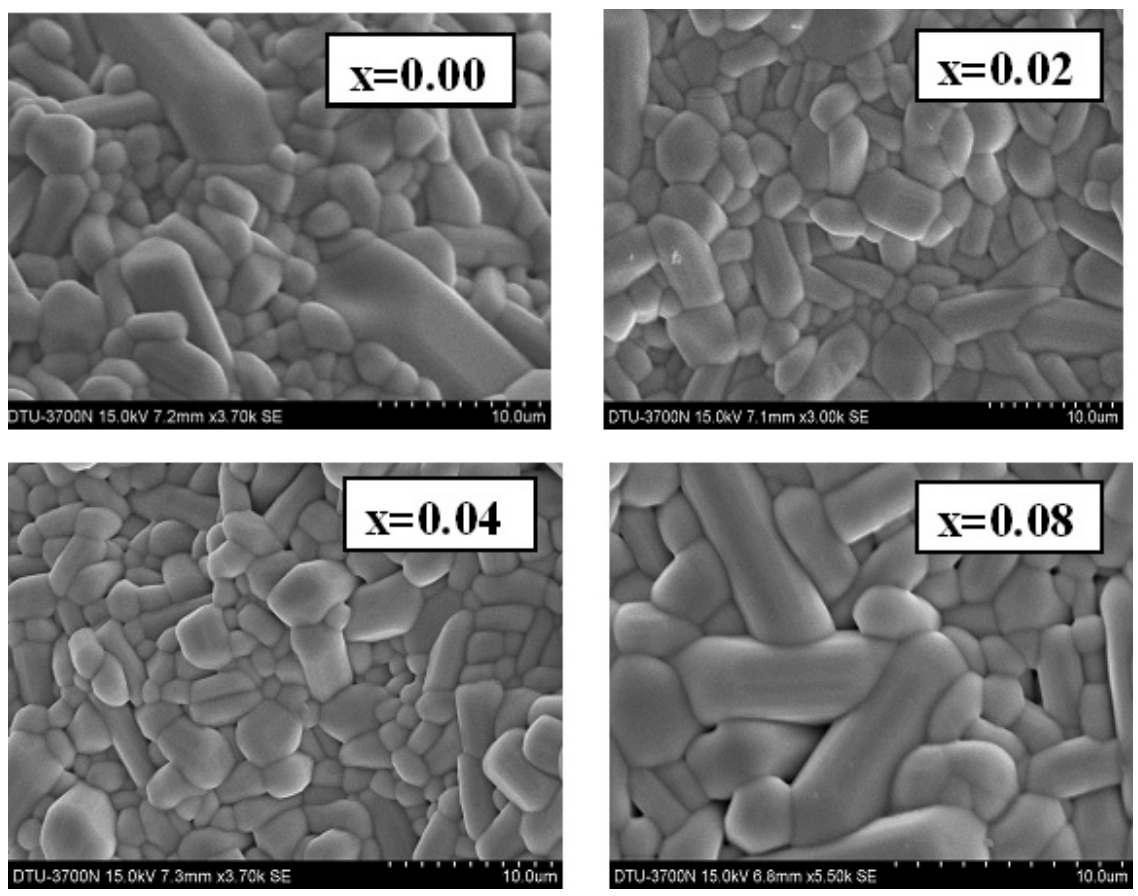

Fig. 2. SEM micrographs for $\mathrm{Ba}_{5} \mathrm{CaTi}_{2-x} \mathrm{Co}_{x} \mathrm{Nb}_{8} \mathrm{O}_{30}$ ceramics. 
increase in temperature and then increased quickly. In addition to this, it was also observed that the dielectric constant increased to larger extent at low frequencies while for higher value of frequency, the increase was observed to be small. It can be correlated to the type of polarization. Interfacial polarization is known to play the most significant role at low frequencies. Interfacial polarizations are due to the increase of charges at the grain boundary. Charge carriers get thermally activated due to the increase in temperature which results in the increase of electron exchange interaction thereby results in an increase of dielectric constant. At high frequencies, electronic and ionic polarizations are the main contributors and their temperature dependence is insignificant. $^{20}$ The dielectric constant was observed to increase up to $x<0.04$ and then a decrease in dielectric constant was observed for $x>0.04$. The increase in the value of dielectric constant is due to thermally activated transport properties, conduction electrons, cations, addition of dopants and due to the presence of space charge. The contribution of space charge is because of the occurrence of oxygen vacancies.

$$
\begin{gathered}
\mathrm{O}_{\mathrm{o}} \leftrightarrow \frac{1}{2} \mathrm{O}_{2}+V_{\mathrm{o}}^{\bullet \bullet}+2 \mathrm{e}^{\prime}, \\
V_{\mathrm{o}}^{\bullet \bullet}+\mathrm{e}^{\prime} \leftrightarrow V_{\mathrm{o}}^{\bullet},
\end{gathered}
$$

where $V_{\mathrm{o}}$ denotes the oxygen vacancies. In addition to the contribution of oxygen vacancies, the value of $\varepsilon_{r}$ also increases due to energetic hopping electron along the direction of applied field between $\mathrm{Nb}^{4+}$ and $\mathrm{Nb}^{5+}$ octahedral sites. $^{21-23}$ With increasing cobalt concentration up to $x=0.04$, dielectric constant decreases and then further increases. It may be attributed due to well-developed grain size as shown in Fig. 2. Also, it is observed that Curie temperature or transition temperature $\left(T_{c}\right)$ increases up to $x=$ 0.04 with increasing cobalt substitution and then decreases with further substitution. This can be understood as: the bond dissociation energy for $\mathrm{Ba}-\mathrm{O}$ bond is $562 \mathrm{~kJ} / \mathrm{mol}$ and the bond dissociation energy for $\mathrm{Ca}-\mathrm{O}$ bond is $464 \mathrm{~kJ} / \mathrm{mol}$. The bond dissociation energy of $\mathrm{Nb}-\mathrm{O}$ is $753 \mathrm{~kJ} / \mathrm{mol}$ which is greater than Co-O $(362 \mathrm{~kJ} / \mathrm{mol})$ and Ti-O $(662 \mathrm{~kJ} / \mathrm{mol}) .{ }^{24}$ So, during high temperature sintering $\mathrm{Co}$, atom starts entering at $\mathrm{Ti}$ site and $\mathrm{Nb}$ due to which the formation of $\mathrm{Co}-\mathrm{O}$ bond
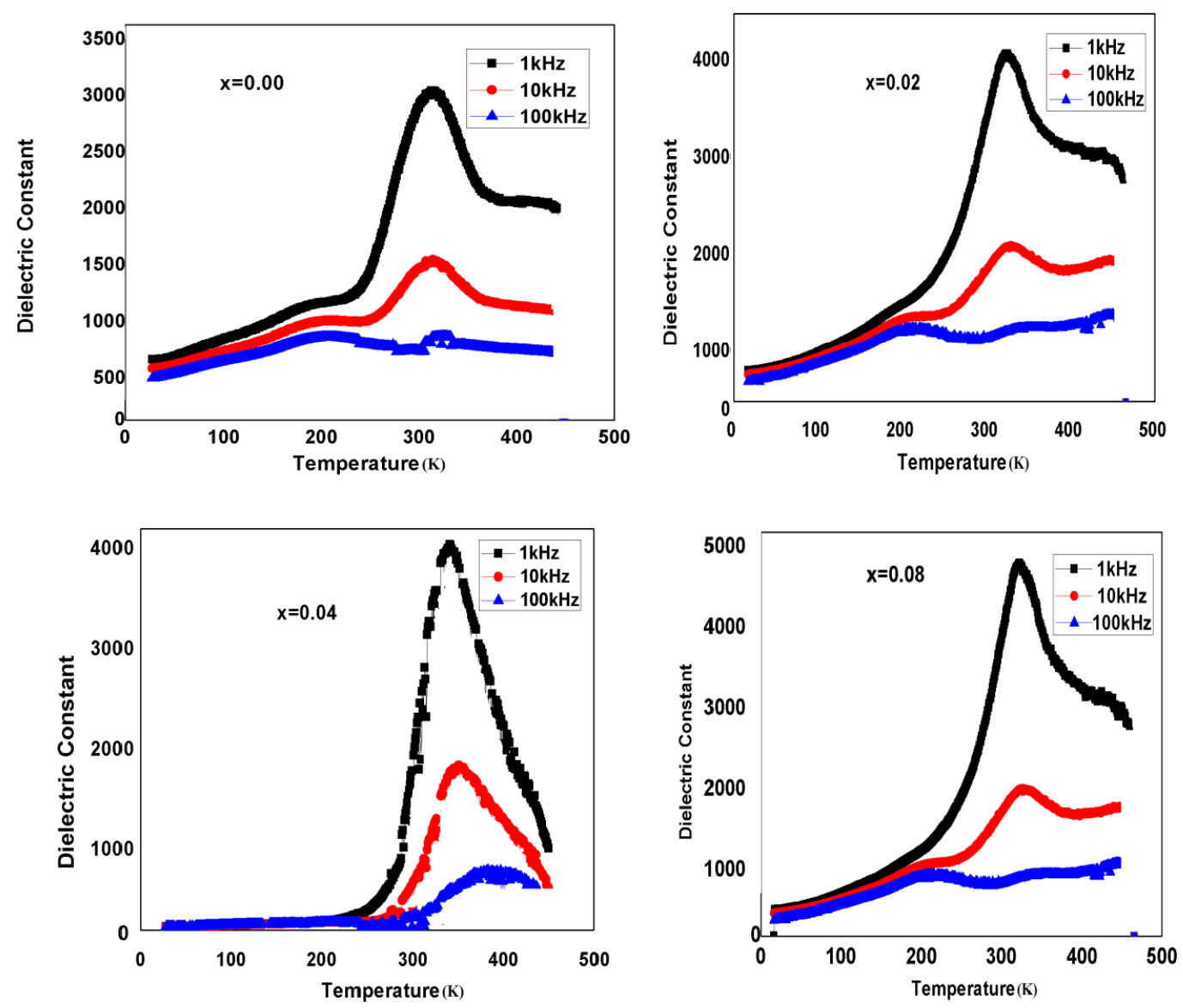

(a)

Fig. 3. (a) Variation of dielectric constant with temperature at different frequencies for $\mathrm{Ba}_{5} \mathrm{CaTi}_{2-x} \mathrm{Co}_{x} \mathrm{Nb}_{8} \mathrm{O}_{30}$ ceramics and (b) variation of dielectric loss tangent with temperature at different frequencies for $\mathrm{Ba}_{5} \mathrm{CaTi}_{2-x} \mathrm{Co}_{x} \mathrm{Nb}_{8} \mathrm{O}_{30}$ ceramics. 

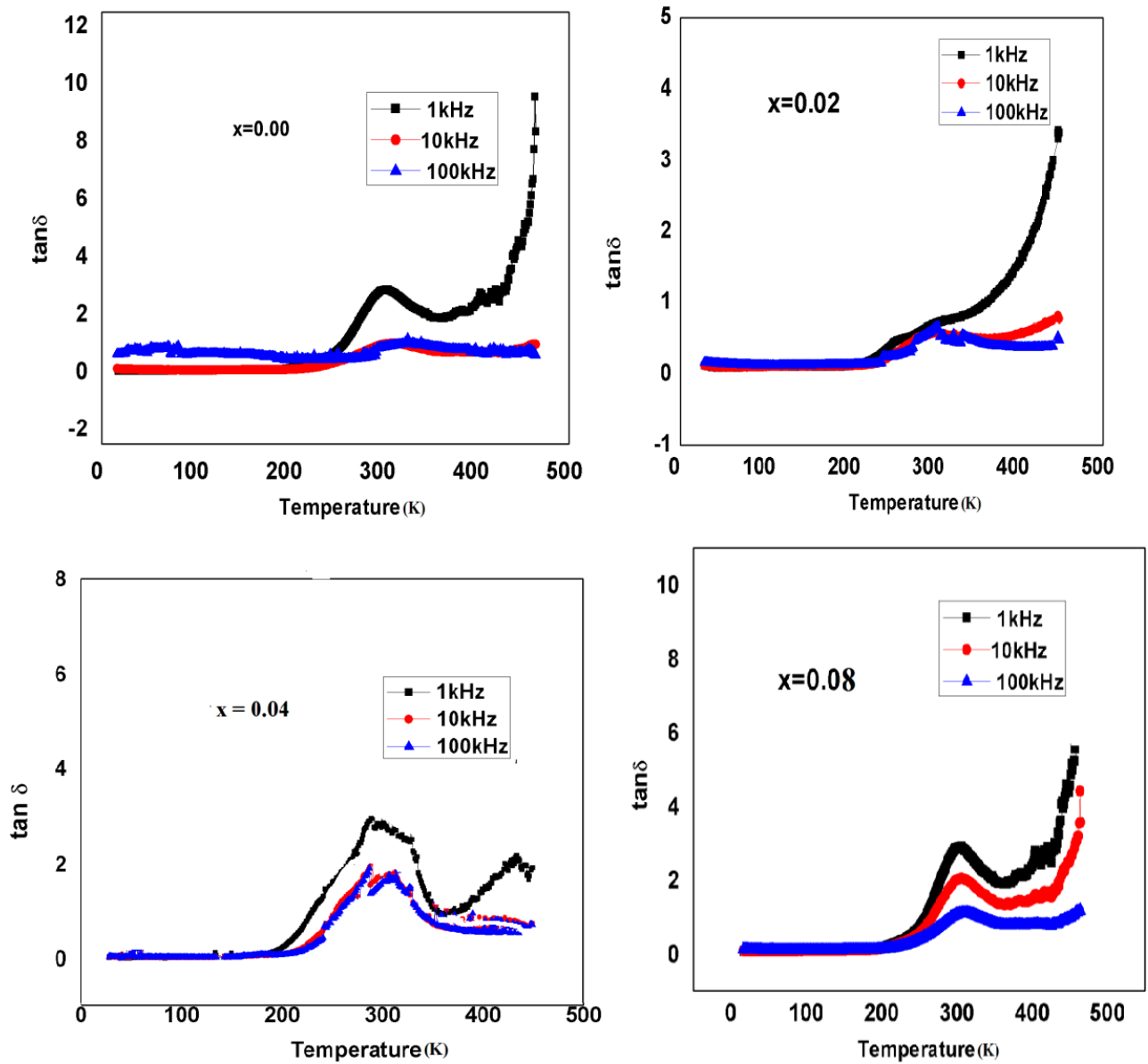

(b)

Fig. 3. (Continued)

takes place. For lower concentration of Co content, $\mathrm{Ba}-\mathrm{O}$ and $\mathrm{Ca}-\mathrm{O}$ bonds are larger and $\mathrm{Ti}-\mathrm{O}$ and $\mathrm{Nb}-\mathrm{O}$ bonds (B-site) are stronger than A site bond and it results in the occurrence of phase transition at higher temperature due to which the transition temperature increases. ${ }^{24-26}$ With further increase of cobalt concentration, the $\mathrm{Co}-\mathrm{O}$ bond increases and this bond becomes stronger than $\mathrm{Ti}-\mathrm{O}$ and $\mathrm{Nb}-\mathrm{O}$ bonds which lead to weak interaction between $\mathrm{BO}_{6}$ octahedra. This results in the decrease of transition temperature $\left(T_{c}\right)$. The variation of dielectric loss of the studied samples from room temperature to $500^{\circ} \mathrm{C}$ is shown in Fig. 3(b). It is observed that the loss is independent of temperature up to $250^{\circ} \mathrm{C}$ at the lower frequency and then increases rapidly with the increase of temperature. ${ }^{27}$ At high temperature region, the sharp increase in dielectric loss may be due to the increased mobile charge carriers arising from defects/vacancies (like oxygen vacancies) in the specimen.

\subsection{Diffuse phase transition}

Figure 3(a) shows the broadening of dielectric peak which may be attributed due to disorder distribution of cations at
A and B sites in TB structure. Diffuse phase transition is frequency-dependent and as temperature increases, broad peak of compound with dielectric constant suggests the ferroelectric-paraelectric phase transition. The degree of disorder or diffusivity $(\gamma)$ can be calculated using the modified Curie-Weiss law of equation: ${ }^{28}$

$$
\ln \left(\frac{1}{\varepsilon}-\frac{1}{\varepsilon_{\max }}\right)=\gamma \ln \left(T-T_{c}\right)+C,
$$

where $\varepsilon$ is the relative permittivity at temperature $T$ and $\varepsilon_{\max }$ is the maximum relative permittivity at temperature $T c, C$ is the curie temperature constant. The parameter $\gamma$ measures the diffuseness of phase transition as $\gamma=1$ corresponds to normal ferroelectric behavior while $1<\gamma<2$ corresponds to diffuse phase transition. ${ }^{29}$ The value of $\gamma$ observed from the fitting curve with transition metal substitution is shown in Fig. 4. It depicts the disorderness in the compound and hence diffuse phase transition is observed with increasing Co content. Parida et $a l^{30}$ have reported the same behavior of TB structure ferroelectric ceramics. 


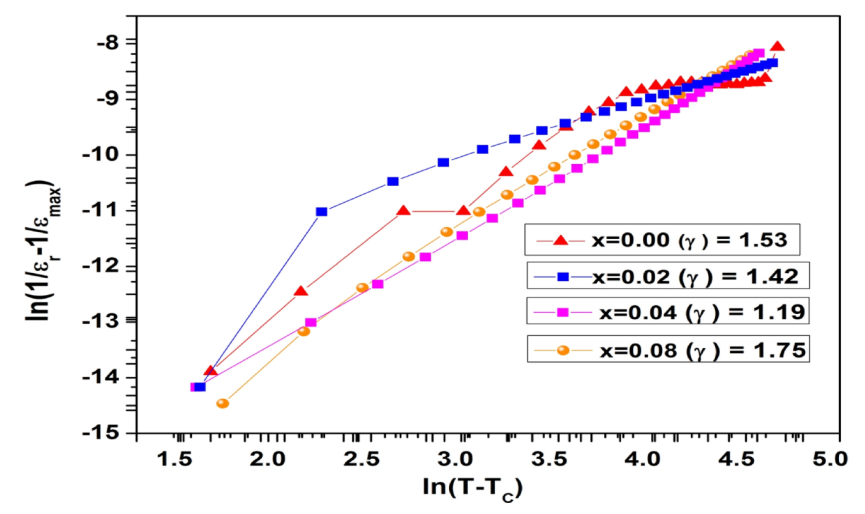

Fig. 4. The plots of $\ln \left(\frac{1}{\varepsilon}-\frac{1}{\varepsilon_{\max }}\right)$ as a function of $\ln \left(T-\mathrm{T}_{c}\right)$ for $\mathrm{Ba}_{5} \mathrm{CaTi}_{2-x} \mathrm{Co}_{x} \mathrm{Nb}_{8} \mathrm{O}_{30}$ ceramics at $10 \mathrm{kHz}$.

\subsection{Ferroelectric study}

The $P-E$ hysteresis loop of $\mathrm{Ba}_{5} \mathrm{CaTi}_{2-x} \mathrm{Co}_{x} \mathrm{Nb}_{8} \mathrm{O}_{30}$ $(x=0.00,0.02,0.04$ and 0.08$)$ ceramics recorded at room temperature is shown in Fig. 5. The hysteresis loop was recorded at a frequency of $50 \mathrm{~Hz}$ and applied field up to $40 \mathrm{kv} / \mathrm{cm}$ which confirmed that the material was lossy and polycrystalline nature as per expectations of Das et al. ${ }^{31}$ It is observed that the remanent polarization $\left(2 P_{r}\right)$ increases for $x \leq 0.04$ and decreases for $x \geq 0.04$ with increasing cobalt concentration because $\mathrm{Co}^{2+}(0.650)$ substitution at $\mathrm{Ti}^{4+}$ (0.605) site results in the lattice shrinkage due to which interspace between oxygen octahedrons inside the unit cell reduces. ${ }^{32-34}$ The shape of hysteresis loop can be correlated to crystalline structure of the materials. It is known that ferroelectric properties are affected by composition, doping, variation in grain size and the presence of defects. ${ }^{35}$ The variation in remanent polarization can also be explained on the basis of grain size. Grain boundary has major effects on polarization. Larger grain size results in small grain boundaries as grain boundaries have high permittivity region, and hence better ferroelectricity. Also, there is polarization discontinuity between grain boundary and bulk material due to which remanent polarization decreases. Coercive field $\left(2 \mathrm{E}_{c}\right)$ of ceramics also strongly depends on crystal imperfection and time

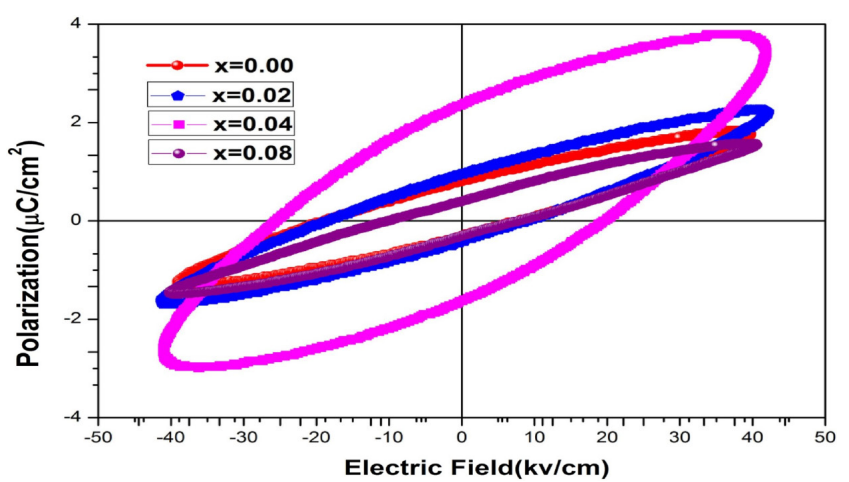

Fig. 5. $P-E$ loops for $\mathrm{Ba}_{5} \mathrm{CaTi}_{2-x} \mathrm{Co}_{x} \mathrm{Nb}_{8} \mathrm{O}_{30}$ ceramics at room temperature. duration of the applied electric field. It is observed that with increasing concentration of Co ions, coercive field decreases. ${ }^{36}$ These results are attributed to the reduction of energy barrier for switching polarization of ferroelectric domains which is easier inside the larger grain as compared to smaller grains.

\subsection{Ferromagnetic study}

Room temperature magnetic study of the synthesized sample was done by using Vibrating Sample Magnetometer VSM with maximum magnetic field 8000 Oe and the $M-H$ plots are shown in Fig. 6. The magnetization of Co-doped multiferroic ceramic was observed to be saturated up to maximum field. The nonsaturated magnetization shows the strong existence of strong antiferromagnetic coupling between adjacent $\mathrm{Co}$ and $\mathrm{Ti}$ mixed ions with ferromagnetic interaction. ${ }^{37}$ The value of remanent magnetization $\left(M_{r}\right)$ and saturation magnetization $\left(M_{s}\right)$ obtained at room temperature is given in Table 2. As perovskite ferroelectric ceramics show a single phase multiferroic by substitution of transition metal $\mathrm{Fe}^{2+}$, $\mathrm{Cr}^{2+}, \mathrm{Mn}^{+}$, etc., the existence of ferromagnetism in Cosubstituted multiferroic ceramic is as per expectation. ${ }^{38,39}$ It can also be explained on the basis of the fact that the substitution of $\mathrm{Co}^{2+}$ ion at $\mathrm{Ti}^{4+}$ site creates oxygen vacancies at octahedral inside the unit cell and also due to suppression of exchange interaction energy at high magnetic field in $M-H$ loop. The variation in remnant and saturation magnetization is due to the magnetic interaction between the adjacent ions. It is well known that in TB multiferroic ceramic exchange

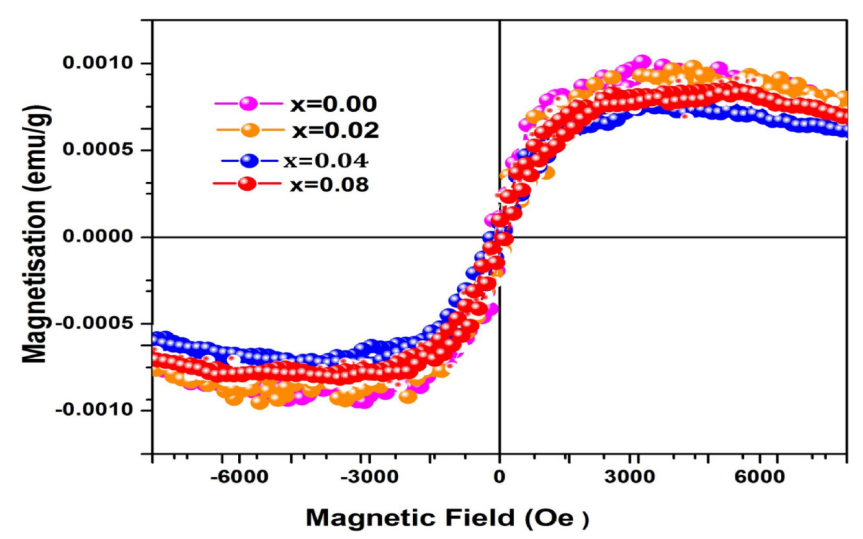

Fig. 6. $M-H$ loops for $\mathrm{Ba}_{5} \mathrm{CaTi}_{2-x} \mathrm{Co}_{x} \mathrm{Nb}_{8} \mathrm{O}_{30}$ ceramics at room temperature.

Table 2. Ferromagnetic parameters for $\mathrm{Ba}_{5} \mathrm{CaTi}_{2-x} \mathrm{Co}_{x} \mathrm{Nb}_{8} \mathrm{O}_{30}$ $(x=0.00,0.02,0.04$ and 0.08$)$ ceramics.

\begin{tabular}{lcc}
\hline Sample (x) & $M_{r} \times 10^{-5}(\mathrm{emu} / \mathrm{g})$ & $M_{s} \times 10^{-4}(\mathrm{emu} / \mathrm{g})$ \\
\hline 0.00 & 7.65 & 7.22 \\
0.02 & 6.04 & 7.32 \\
0.04 & 5.72 & 7.20 \\
0.08 & 5.48 & 8.35 \\
\hline
\end{tabular}


interaction mechanism is due to $\mathrm{A}$ site and $\mathrm{B}$ site sublattice ions. The $\mathrm{A}-\mathrm{A}$ and $\mathrm{B}-\mathrm{B}$ interaction between lattices is very weak, but interaction of A-B is the strongest, it may align all the magnetic spins at $\mathrm{A}$ site in one direction and those at $\mathrm{B}$ site in the opposite direction, results oppositely and saturated magnetized sublattice at $0 \mathrm{~K}$. Hence, net saturation magnetization is given by following equation:

$$
M_{s}=M_{\mathrm{B}}-M_{\mathrm{A}}
$$

In ceramics, magnetic ion favoritism in A-B site interaction depends on temperature. Generally, few sites favor certain ions at low temperature and other ions favor at high temperature. ${ }^{40-42}$ Here, samples are prepared under high sintering condition due to which few cobalt ions are expected to go into tetrahedral site and more than half goes to octahedral site (B). This gives magnetic moment to Cobalt ions. As the titanium ion is substituted by the transition cobalt ion, octahedral site (B) energy is large for Co ions as compared to A site ions.

\section{Conclusion}

In summary, the specimen $\mathrm{Ba}_{5} \mathrm{CaTi}_{2-x} \mathrm{Co}_{x} \mathrm{Nb}_{8} \mathrm{O}_{30}$ with different copper concentrations was synthesized by solid state reaction method. X-ray diffractograms show that the specimens have single phase tetragonal structure at room temperature. The SEM depicted the presence of well-developed grain with uniform grains. The co-relation between $P-E$ and $M-H$ loops is observed. Diffuse phase transition is observed in the studied samples. The dielectric constant and remnant polarization were found to be higher at $x=0.04$ and the specimen was observed to be suitable for microwave tunable devices and infrared detector applications.

\section{Acknowledgment}

Author K. M. Batoo is thankful to the Deanship of Scientific Research at King Saud University for its funding through the Research Group Project No. RG-1437-030.

\section{References}

${ }^{1}$ S. Jindal, A. Vasishth, S. Devi and G. Anand, A review on tungsten bronze ferroelectric ceramics as electrically tunable devices, Integrated Ferroelectrics 186(1), 1 (2018).

${ }^{2} \mathrm{G}$. Busch, Early history of ferroelectricity, Ferroelectrics 74(1), 267 (1987).

${ }^{3}$ F. Jona and G. Shirane, Ferroelectric Crystals, Vol. 1 (Pergamon, New York, 1962).

${ }^{4}$ M. E. Lines and A. M. Glass, Principles and Applications of Ferroelectrics and Related Materials (Oxford University Press, Oxford, 1977).

${ }^{5}$ S. Jindal, A. Vasishth, S. Devi and B. Singh, Synthesis and characterization of polycrystalline $\mathrm{Ba}_{5} \mathrm{CaTi}_{2-x} \mathrm{M}_{x} \mathrm{Nb}_{8} \mathrm{O}_{30}(M=\mathrm{Cu})$ tungsten bronze electro ceramics, Ferroelectrics 519(1), 9 (2017).
${ }^{6}$ P. Ganguly, S. Devi, A. K. Jha and K. L. Deori, Dielectric and pyroelectric studies of Tungsten-Bronze structured Ba5SmTi3Nb7O30 ferroelectric ceramics, Ferroelectrics 387(1), 111 (2009).

${ }^{7}$ J.-F. Li et al., Piezoelectric properties of sol-gel-derived ferroelectric and antiferroelectric thin layers, J. Appl. Phys. 75(1), 442 (1994).

${ }^{8}$ J. Ravez and A. Simon, Temperature and frequency dielectric response of ferroelectric ceramics with composition $\mathrm{Ba}(\mathrm{Ti} 1-x \mathrm{Zr} x$ ) O 3, Eur. J. Solid State Inorg. Chem. 34(11), 1199 (1997).

${ }^{9}$ B. Behera, P. Nayak and R. N. P. Choudhary, Structural, dielectric and electrical properties of LiBa $2 X 5 \mathrm{O} 15(X=\mathrm{Nb}$ and $\mathrm{Ta})$ ceramics, Mater. Chem. Phys. 100(1), 138 (2006).

${ }^{10}$ K. Kathayat, A. Panigrahi, A. Pandey and S. Kar, Effect of Holmium doping in Ba5RTi3V7O30 ( $\mathrm{R}=$ rare earth element) compound, Integr. Ferroelectr. 118(1), 8 (2010).

${ }^{11}$ P. R. Das, B. Pati, B. C. Sutar and R. N. P. Choudhury, Study of structural and electrical properties of a new type of complex tungsten bronze electroceramics; Li2Pb2Y2W2Ti4V4O30, J. Mod. Phys. 3(8), 870 (2012).

${ }^{12} \mathrm{P}$. Ganguly and A. K. Jha, Structural and electrical properties of Ba 5-x Ca $x$ SmTi 3 Nb 7 O $30(x=0-5)$ ferroelectric ceramics, J. Alloys Compd. 495(1), 7 (2010).

${ }^{13}$ W. Chen, W. Z. Yang, X. Q. Liu and X. M. Chen, Structural, dielectric and magnetic properties of $\mathrm{Ba} 3 \mathrm{SrLn} 2 \mathrm{Fe} 2 \mathrm{Nb} 8 \mathrm{O} 30$ $(\mathrm{Ln}=\mathrm{La}, \mathrm{Nd}, \mathrm{Sm})$ filled tungsten bronze ceramics, J. Alloys Compd. 675, 311 (2016).

${ }^{14}$ M. Yan, Y. Q. Tan, H. Zhao, J. Peng, X. L. Xiao and Z.-B. Hu, Crystal structure, dielectric and magnetic properties of $\mathrm{Ba} 5 \mathrm{NdNi}$ $1.5 \mathrm{Nb} 8.5$ O 30 tungsten bronze ceramic, Mater. Chem. Phys. 136(2), 487 (2012).

${ }^{15}$ X. L. Zhu, X. Q. Liu and X. M. Chen, Crystal structure and dielectric properties of Sr5RTi3Nb7O30 $(\mathrm{R}=\mathrm{La}, \mathrm{Nd}, \mathrm{Sm}$, and Eu) tungsten bronze ceramics, J. Am. Ceram. Soc. 94(6), 1829 (2011)

${ }^{16}$ P. Ganguly, A. K. Jha and K. L. Deori, Investigations of dielectric, pyroelectric and electrical properties of $\mathrm{Ba} 5 \mathrm{SmTi} 3 \mathrm{Nb} 7 \mathrm{O} 30$ ferroelectric ceramic, J. Alloys Compd. 484(1), 40 (2009).

${ }^{17}$ N. Wakiya, J.-K. Wang, A. Saiki, K. Shinozaki and N. Mizutani, Synthesis and dielectric properties of Ba $1-x \mathrm{R} 2 x / 3 \mathrm{Nb} 2 \mathrm{O} 6$ (R: rare earth) with tetragonal tungsten bronze structure, J. Eur. Ceram. Soc. 19(6) 1071 (1999).

${ }^{18}$ S. Jindal, A. Vasishth and S. Devi, Structural and dielectric properties of Co substituted multi-ferroic ceramic, J. Basic Appl. Eng. 2(21), 1877 (2015).

${ }^{19}$ P. A. Jha and A. K. Jha, Influence of processing conditions on the grain growth and electrical properties of barium zirconate titanate ferroelectric ceramics, J. Alloys Compd. 513, 580 (2012).

${ }^{20} \mathrm{~N}$. Setter and L. E. Cross, The role of B-site cation disorder in diffuse phase transition behavior of perovskite ferroelectrics, J. Appl. Phys. 51(8), 4356 (1980).

${ }^{21}$ N. Sawangwan, J. Barrel, K. Mackenzie and T. Tunkasiri, The effect of $\mathrm{Zr}$ content on electrical properties of $\mathrm{Ba}$ (Ti 1-x $\mathrm{Zr}$ x) $\mathrm{O} 3$ ceramics, Appl. Phys. A, Mater. Sci. Process. 90(4), 723 (2008).

${ }^{22}$ S. K. Rout, E. Sinha and S. Panigrahi, Dielectric properties and diffuse phase transition in $\mathrm{Ba} 1-x \mathrm{Mg} x$ Ti $0.6 \mathrm{Zr} 0.4 \mathrm{O} 3$ solid solutions, Mater. Chem. Phys. 101(2), 428 (2007).

${ }^{23}$ P. B. Jamieson, S. C. Abrahams and J. L. Bernstein, Ferroelectric tungsten bronze-type crystal structures. II. Barium sodium niobate 
$\mathrm{Ba}(4+x) \mathrm{Na}(2-2 x) \mathrm{Nb} 10 \mathrm{O} 30$, J. Chem. Phys. 50(10), 4352 (1969).

${ }^{24}$ S. C. Abrahams, P. B. Jamieson and J. L. Bernstein, Ferroelectric tungsten bronze-type crystal structures. III. potassium lithium niobate $\mathrm{K}(6-x-y) \mathrm{Li}(4+x) \mathrm{Nb}(10+y) \mathrm{O} 30$, J. Chem. Phys. 54(6) 2355 (1971).

${ }^{25}$ M. S. Kim, C. D. Oh, J. H. Lee, J. J. Kim, H. Y. Lee and S. H. Cho, Microstructure evolution and electrical properties of $\mathrm{Ba} 2 \mathrm{NaNb} 5 \mathrm{O} 15$ and $\mathrm{Pb}(\mathrm{Mg} 1 / 3 \mathrm{Nb} 2 / 3) \mathrm{O} 3$ composites, Ferroelectrics 334(1), 105 (2006).

${ }^{26}$ X. Zong, Z. Yang, H. Li and M. Yuan, Effects of WO 3 addition on the structure and electrical properties of $\mathrm{Pb} 3 \mathrm{O} 4$ modified PZTPFW-PMN piezoelectric ceramics, Mater. Res. Bull. 41(8) 1447 (2006).

${ }^{27}$ S. Sen and R. N. P. Choudhary, Studies of structural and electrical properties of Ba $1-x$ Ca $x$ Sn 0.05 Ti $0.95 \mathrm{O} 3$, ferroelectric ceramics, Mater. Lett. 58(5), 661 (2004).

${ }^{28}$ Y. Takahashi, On the origin of the Curie-Weiss law of the magnetic susceptibility in itinerant electron ferromagnetism, J. Phys. Soc. Jpn. 55(10), 3553 (1986).

${ }^{29}$ P. Ganguly and A. K. Jha, Structural, dielectric and electrical properties of $\mathrm{CaBa} 4 \mathrm{SmTi} 3 \mathrm{Nb} 7 \mathrm{O} 30$ ferroelectric ceramic, Bull. Mater. Sci. 34(4), 907 (2011).

${ }^{30}$ B. N. Parida, P. R. Das, R. Padhee and R. N. P. Choudhary, Phase transition and conduction mechanism of rare earth-based tungstenbronze compounds, J. Alloys Compd. 540, 267 (2012).

${ }^{31}$ D. K. Pradhan, B. Behera and P. R. Das, Studies of dielectric and electrical properties of a new type of complex tungsten bronze electroceramics, J. Mater. Sci, Mater. Electron. 23(3), 79 (2012).

${ }^{32}$ M. X. Cao, X. L. Zhu, X. Q. Liu and X. M. Chen, Crystal structure and ferroelectric behaviors of $\mathrm{Ba}_{5} \mathrm{SmTi}_{3} \mathrm{Ta}_{7} \mathrm{O}_{30}$ and $\mathrm{Ba}_{4} \mathrm{Sm}_{2} \mathrm{Ti}_{4} \mathrm{Ta}_{6} \mathrm{O}_{30}$ tungsten bronze ceramics, J. Am. Ceram. Soc. 93(3), 782 (2010).

${ }^{33}$ G. Gong, G. Zerihun, Y. Fang, S. Huang, C. Yin and S. Yuan, Relaxor behavior and large room-temperature polarization of ferroelectric Sr4CaBiTi3Nb7O30 ceramics, J. Am. Ceram. Soc. 98(1), 109 (2015).

${ }^{34}$ J. Hao, W. Bai, W. Li and J. Zhai, Correlation between the microstructure and electrical properties in high-performance $(\mathrm{Ba} 0$. 85Ca0. 15)( $\mathrm{Zr} 0$. 1Ti0. 9) O3 lead-free piezoelectric ceramics, J. Am. Ceram. Soc. 95(6), 1998 (2012).

${ }^{35}$ S. Devi and A. K. Jha, Enhancement of piezoelectric and ferroelectric properties in wolframium substituted barium titanate ferroelectric ceramics, Indian J. Phys. 86(4), 279 (2012).

${ }^{36}$ Q. Xu, X. Chen, W. Chen, S. Chen, B. Kim and J. Lee, Synthesis, ferroelectric and piezoelectric properties of some (Na 0.5 Bi 0.5) TiO 3 system compositions, Mater. Lett. 59(19), 2437 (2005).

${ }^{37}$ X. L. Zhu, X. M. Chen, X. Q. Liu and X. G. Li, Ferroelectric phase transition and low-temperature structure fluctuations in $\mathrm{Ba}_{4} \mathrm{Nd}_{2} \mathrm{Ti}_{4} \mathrm{Nb}_{6} \mathrm{O}_{30}$ tungsten bronze ceramics, J. Appl. Phys. 105, 124110 (2009).

${ }^{38}$ P. P. Liu, S. Y. Wu, X. L. Zhu, X. M. Chen and X. Q. Liu, Structure, dielectric and magnetic properties of $\mathrm{Ba}_{6} \mathrm{FeNb}_{9} \mathrm{O} 30$ tungsten bronze ceramics, J. Mater. Sci., Mater. Electron. 22(7), 866 (2011).

${ }^{39}$ H. Deng, M. Zhang, Z. Hu, Q. Xie, Q. Zhong, J. Wei and H. Yan, Enhanced dielectric and ferroelectric properties of $\mathrm{Ba}$ and Ti co-doped BiFeO3 multiferroic ceramics, J. Alloys Compd 582, 273 (2014).

${ }^{40}$ M. C. Foster, G. R. Brown, R. M. Nielson and S. C. Abrahams, Ba6CoNb9O30 and Ba6FeNb9O30: Two new tungsten-bronze-type ferroelectrics. Centrosymmetry of Ba5. 2K0. 8U2. 4Nb7. $6 \mathrm{O} 30$ at 300 K, J. Appl. Crystallogr. 30(4), 495 (1997).

${ }^{41}$ X. Qi, and J.-Y. Chang, Single crystal growth and characterisations of transition metal ion doped TTB ferroelectrics, Ferroelectrics 421(1), 60 (2011).

${ }^{42}$ S. Kumar, R. Kumar, P. Thakur, K. H. Chae, B. Angadi and W. K. Choi, Electrical transport, magnetic, and electronic structure studies of $\mathrm{Mg} 0$. 95Mn0. 05Fe2-2xTi2 $x \mathrm{O} 4 \pm \delta \quad(0 \leq x \leq 0.5)$ ferrites, J. Phys., Condens. Matter 19(47), 476210 (2007). 